\title{
Probiotics: past, present and future challenges
}

\author{
Baraa M. AL-Taha1 , Jamal Wadi ${ }^{2}$, Asem A. Shehabi ${ }^{1}$
}

\section{Abstract}

The term probiotic is utilized to describe useful living microorganisms, intended to colonize the large intestine, when consumed in adequate amounts, they confer physiological health benefits to the host. In the last decade, probiotics have been widely used as a nutritional supplement and became a rapidly developing research topic that gained importance in medicine. Recent clinical trials have found that consumption of beneficial bacterial species can manipulate gut flora and reduce or prevent the development of certain serious diseases. This brief review article is intended to discuss the status of research on probiotics, and the value of their consumption in human health.

\section{General description of probiotics}

Microorganisms may be arbitrarily subdivided for human health into two major groups; the pathogenic and the beneficial groups. Microorganisms which benefit humans have been detected for thousands of years through the process of using them in food and alcohol fermentations. In the 1900s, a Russian scientist; Élie Metchnikoff (Nobel prize winner) linked the consumption of the fermented milk produced by the Bulgarians to their long and healthy lives. In 1930, the first clinical trial on probiotics outcome in human constipation was completed [1]. A Japanese microbiologist, Minoru Shirota in 1930, stated that "many diseases could be prevented if human beings have the right combination of bacteria in their gut". He has proved successfully by experiment that a beneficial bacterial strain when it was given alive and in large quantity can improve intestinal functions and consequently the host health. Therefore, he introduced a beneficial bacterial strain (Lactobacillus casei strain Shirota) to make a fermented milk drink called (Yakult) for consumption as a healthy food. Currently, Yakult is a company that have a variety of fermented food products and considered a global leader in probiotic market [2].
1 School of Medicine, Department of Pathology Microbiology, The Jordan University, Amman, Jordan.

2 School of Medicine, Department of Internal Medicine/Division of Infectious diseases, Jordan University Hospital, Amman, Jordan

\section{Contact information:}

Prof. Dr. Asem A. Shehabi.

” asashehabi2@gmail.com

\section{Keywords}

Probiotics; Health Benefit; Side Effects. 
The World Health Organizations (WHO) and Food and Agriculture Organization (FAO) describe probiotics as live microorganisms that have beneficial properties for the host when ingested in adequate amounts and confer health benefits [2]. FAO/WHO expert group also made guidelines for probiotics evaluation that contain information needed to verify health claims [3]. Nowadays, probiotics are a multibillion dollars global industry, and they are used intensively in medical research and treatment [4]. Nevertheless, it is necessary to differentiate between the term probiotic, prebiotic, and symbiotic. Prebiotic is defined as non-digestible food ingredients that affect the host by selectively stimulating the growth and activity, or both for a limited number of beneficial bacteria in the intestine, whereas the mixture of prebiotics and probiotics is referred to as symbiotic which is expected to induce synergism in the host intestine [3]. WHO and FAO recommend that the term "symbiotic" be used only if the net health benefit is synergistic [4].

\section{The impact of probiotics}

Probiotics are being used to promote several health benefits and to relief from many gastrointestinal and other diseases. This development has occurred because probiotics may help in digestion, production of beneficial products, stimulating the immune response, competing with pathogens and producing bactericides during their presence in the host $[2,5]$. Several intestinal diseases may develop following an infection with the enteric pathogens or the overgrowth of one bacterial type. These conditions could be controlled or prevented once we control the balance of intestinal microflora $[6,7]$. Probiotics are considered safe because they have been consumed for a long time without observing serious side effects. The main reason behind their suggested safety is the long-term consumption as food before they were introduced in medicine $[9,10]$. Theoretically, probiotics could result in some adverse effects; including the contribution to antibiotics resistance through their ability to transfer resistance genes to human intestinal flora, overstimulation of the immune system and producing adverse metabolic activity [10]. The most common microorganisms used in the preparation of probiotics are those related to human gut flora. These include primarily lactic acid bacteria (LAB), Bifidobacteria spp. and Propionibacteria spp. These are frequently found in fermented foods, particularly dairy products. They are quantitatively the most essential ingested bacteria. Therefore, most studies focused on examining the impact of these bacterial groups in probiotics on the gut microbiota and the host health $[10,11]$.

\section{Probiotics mechanisms of actions}

The exact mechanisms by which probiotics exert their effects are incompletely understood, some mechanisms were proposed; some probiotics excrete lactase that assist in lactose digestion [1]. Probiotics can produce lactic acid, benzoic acid and hydrogen peroxide altering gut $\mathrm{pH}$ and these products have protective effects mostly against acid-sensitive bacteria [7]. Probiotics may restore the composition of gut microbiome and introduce beneficial functions to the gut microbes, resulting in amelioration or prevention of gut inflammation [12]. Some probiotics create nutrients like provitamins, amino acids, polyamines, antioxidants, growth factors and short-chain fatty acids such as acetic acid which has antimicrobial function [2, 7]. Probiotics have some positive effects on both specific and non-specific immune response of the host by stimulating natural killer cells, monocytes, neutrophils and macrophages. This feature could be a result of probiotics ability to attach to gut epithelium, since attachment of normal and beneficial bacteria was found to prevent the immune system over-stimulation which could be brought by pathogenic bacteria [7]. Other effects of probiotics include the reduction of the concentration of cancer-promoting enzymes [5], prevention and alleviation of nonspecific irregular complaints of 
the gastrointestinal tracts in healthy people [5]. Additionally, the possibility of increasing the mucosal immune system by inducing protective cytokines like IL-10 and TGF-beta and immune-modulator cells, while inhibiting the pro-inflammatory factors like fecal tumor necrosis factor-alpha which mediates intestinal inflammation is suggested [5, 12]. Also, probiotics can compete with pathogenic bacteria by different mechanisms such as competing with the pathogenic microorganisms for food and growth factors, production of antimicrobial compounds like cytokines and butyric acid, and probiotics aid to increase mucosal renewal and upregulation of mucin genes in intestine, this can enhance mucous generation which will inhibit the adherence and colonization of many foreign microorganisms [1, 7, 12].

\section{The Benefits of probiotics}

In recent years, the natural products have experienced rapid changing and attracted increasing public attention for those who are looking for better health. There are several reasons behind this health care interest, including foundation of many health promoting organizations, the wide range of health information available on the internet, and the increasing interest in the natural remedies. Probiotics consumption is one of the issues that gained increased interest and ongoing research [13].

\section{Gastrointestinal flora}

The effect of the human flora on gastrointestinal tract (GIT) is important for the proper functioning of the digestive system and the maintenance of good health. It is estimated that the intestinal microflora consists of 500-1000 distinctive species of bacteria in a single person, approximately their population is 10 times the number of the host body cells [14]. In addition, the human intestinal tract may transiently contain a variety of viruses, yeast and parasites [15]. Humans acquire the GIT flora during the delivery process as they descend the birth canal, then, from their surrounding environment. Escherichia coli and Streptococcus species are the first to colonize the GI tract, later it will be followed by Bifidobacteria, Staphylococci, Lactobacilli, Micrococci, and Propionibacteria. Over time, microflora shift to anaerobic bacteria types, with some interindividual variations. The predominant bacteria in the human gut become mostly composed of Bacteroides, Firmicutes and to less amount of Proteobacteria, Actinobacteria, Fusobacteria, and Verrucobacteria $[14,15]$. It was reported that the human GIT maintains a balance between the microflora and the immune system, when the intestinal microflora is altered or modified. A variety of diseases may developed especially diarrhea initiated by infectious agent, antibiotic-associated or traveler's diarrhea, and constipation, gastroenteritis, inflammatory gut disease, irritable bowel syndrome, allergy and some types of intestinal cancers [17]. For example, over-growth of one bacterial species may alter the digestive function, affecting the intestinal immunological function and induce disturbance of beneficial characteristics of other bacterial species [5]. The following major health benefits have been reported to be associated with probiotics treatment:

\section{Diarrhea}

Acute diarrheas may range between 2 to 4 days in $59 \%$ of persons, causing the loss of ample amounts of fluids especially in children and the elderly patients [18]. The use of probiotics is proved to be effective against travelers' diarrhea and other infectious diarrheas including viral causes e.g. rotaviruses, reducing the frequency and duration. Also, probiotics were found to reduce the rates of antibiotics associated diarrhea that may occur in about 5 $-25 \%$ of adult and young patients during antibiotics treatment, but patients must receive high dose of probiotics since low doses has no significant effect [17-21]. 


\section{Constipation}

Constipation as defined "less than three defecations per week and / or hard stool", it is a common digestive problem among population in all age groups, mostly increased in the elderly, due to the changes in dietary habits and reduced physical activity. Probiotics were found effective in decreasing the severity of constipation. Multispecies probiotics have improved defecation frequency in pregnant women with constipation. Lactobacillus reuteri have a remarkable effect against constipation in adults, pregnant women and children. Bifidobacterium tetragenous was found safe and effective for ameliorating constipation, even in cancer patients. Therefore, probiotics can be effective and safe in the treatment of constipation in cancer patients [22, 23, 24].

\section{Helicobacter pylori infections}

H. pylori is gram-negative spiral bacteria,-frequently inhabit the human stomach and duodenum. It is commonly associated with peptic ulcer disease, gastritis, adenocarcinoma and Mucosa-Associated Lymphoid Tissue (MALT lymphoma). The standard therapy prescribed to eradicate the infection is composed of two antibiotics and a proton pump inhibitor. However, bacterial resistance and drug regimen adverse effects compromises their effectiveness, reducing $H$. pylori eradication to less than 55\%. A recent metanalysis by Haghdoost et al. (2017) evaluated the impact of probiotics addition to the standard triple therapy in the quest of eradicating $H$. pylori infection, their findings suggested probiotics benefit regarding $H$. pylori eradication in all age groups, when added to the standard triple therapy [25]. Furthermore, probiotics helped in the triple therapy tolerance when minimized nausea, vomiting, diarrhea, and epigastric pain-and improving patients' compliance. Lactobacillus salivarius produces lactic acid which was shown to be effective in inhibiting the growth of $\mathrm{H}$. pylori and reducing urease activity, an enzyme essential to maintain $H$. pylori colonization and infection. The addition of the probiotic adds about $10 \%$ efficacy to the eradication rate. This is substantial as the prevalence of $\mathrm{H}$. Pylori infection is high and can reach around $40 \%$ in the developed countries $[25,26]$.

\section{Inflammatory bowel diseases (IBD)}

Ulcerative colitis and Crohn's disease are idiopathic in etiology, but are associated with abnormal intestinal flora and malfunctioning of mucosal barrier [2]. It was found that there is a remarkable decrease in the microflora in the IBD patients, mostly, the antiinflammatory bacteria Faecalibacterium prausnitzii compared with healthy controls. Moreover, it was noticed that there was a difference in the microflora between the relapses and remissions phases of the disease when patients with IBD were treated with probiotics [14].

\section{Ulcerative colitis (UC)}

It is characterized by inflammation and injury of the colonic mucosa with relapses and remissions. Steroids are commonly used to induce a remission in moderate to severe UC patients. Some patients become resistant to steroids treatment or may develop adverse reactions. Probiotics treatment has shown to prevent remissions in more than $10 \%$ of patients during 12 months follow up period compared with placebo-treated patients [14, 28].

\section{Crohn's disease (CD)}

It is a chronic, lifelong condition. Chronic inflammation may be found in patients' GIT anywhere from the mouth to the anus, but commonly affects the end of the small intestine and the beginning of the colon. The cause of Crohn's disease is still not well established. However, intestinal immune system and bacterial antigens originated from intestinal bacterial flora, or infection are incriminated to trigger such chronic inflammatory condition [29]. Many studies using single bacterial species of probiotics showed no effectiveness in preventing the recurrence of $C D$ after surgical intervention, howe- 
ver, one-year consumption had noteworthy results of reducing recurrence as well as the pro-inflammatory cytokines, especially when probiotics treatment is administered before surgery rather than later [29]. Several studies revealed controversial results; two studies were conducted using Lactobacillus GG, the first one demonstrated a considerable clinical improvement in children suffering from active CD, while the other study found that the probiotics cannot prevent its relapse, with no significant statistical differences compared with placebo [30]. Whereas, a study used Escherichia coli in high dose has reported a significant effect in the prevention of relapses in $C D$ patients, and the use of a mixture of eight bacterial strains did not have a significant result over placebo after 90 days consumption for the prevention of CD recurrence [30].

\section{Lactose intolerance}

Lactose intolerance is a clinical ailment that results from lactose mal-digestion which develops following the decreasing lactase enzyme activity in human intestine, this enzyme is responsible for digestion of lactose (disaccharide composed of glucose and galactose) which is usually found in mammalian milk. Undigested lactose in the large intestine will activate the action of microflora to facilitate its fermentation, with the production of hydrogen, carbon dioxide and organic acids [27]. This is clinically manifested as diarrhea, vomiting, bloating, abdominal pain, flatulence and cramps. The primary intervention against lactose intolerance is to limit the use of the diary product, hence, limiting their benefits, other interventions is to use lactase enzyme supplements. The use of probiotics, especially lactic acid-producing bacteria is useful to metabolize and absorb lactose, and decrease the fermentation products due to its ability to produce lactase enzyme. The use of Lactobacillus acidophilus can also improve lactose intolerance symptoms during lactose consumption $[27,26]$.

\section{Probiotics and immune system}

The use of probiotics enhances the immune system by contributing with specific and nonspecific immune responses especially cytokines release, natural killer, macrophage activation, increase immunoglobulin levels. This immune reactions occurs without a noticeable harmful inflammatory response [31, 32]. Lactobacillus and Bifidobacterium species are the most known bacteria included in such probiotics. It seems that the types and the doses of the bacterial strains are significant factors that may influence the host immune system [32]. Some Lactobacillus strains have shown to affect many aspects of the immune system and to suppress the inflammation associated with atherosclerosis, whereas, other strains of the same species like Lactobacillus species have proatherogenic role [31].

Several studies showed that probiotics administered as supplements improved the immune system and could be administrated to neonates and the elderly. The daily use of the probiotics Lactobacillus rhamnosus HNO01 and Bifidobacterium lactis by the mother before delivery and during lactation showed significant increase in neonate cord blood cytokine (IFN- $\gamma$ ) as well as in IgA in breast milk [32]. A recent study showed a significant increase in phagocytic activity of blood leukocytes after three weeks of milk probiotics consumption or the consumption of probiotics-supplemented milk for nine weeks by healthy elderly persons. Also, it showed a significant increase of T lymphocytes and Natural Killer [32].

\section{The use of probiotics as adjuvants}

The use of probiotics to improve the immunity after vaccination has been studied and indicated meaningful results. A recent review article reported by Zimmermann and Curtis found that among 26 studies involved 3812 participants, and investigating the effect of 40 different probiotic strains on the responses for 17 different vaccines, a beneficial effect of probiotics was reported in about half of the studies. The evidence for a beneficial effect of 
probiotics on vaccines responses was strongest for oral vaccination, and for parenteral influenza vaccination. Though, substantial variations between studies in the choice of probiotic, strain, dose, viability, purity, and duration and timing of administration were reported. Their review suggested that probiotics offer a relatively inexpensive intervention to improve vaccine efficacy and duration of protection. Future studies must focus on establishing optimal strains, doses and timing of administration in relation to vaccination [33].

The use of Lactobacillus casei to motivate the immune response for influenza vaccination did not show a significant effect through an increase of anti-influenza antibody, however, the duration of the upper respiratory symptoms was reduced [34]. Lactobacillus plantarum had improved the immune response to influenza vaccine in elderly by increasing influenza specific IgA and IgG [35]. LactobaciIlus paracasei have significantly increased both the influenza-specific $\lg G$ and $\lg A$ after its consumption two weeks before and 4 weeks after vaccination, while no difference in the cytokines level was detected [36]. The administration of Lactobacillus rhamnosus and Lactobacillus paracasei in association of live attenuated poliovirus vaccination have shown effectiveness by increasing the poliovirus-specific $\lg G$ and $\lg A$ [32]. The combination of probiotic of Lactobacillus casei with oral rotavirus vaccine indicated a significant increase in $\lg \mathrm{M}$ and $\lg \mathrm{A}$ compared with placebo [37].

\section{Probiotics for allergy prevention}

The prevalence of allergic diseases and asthma are increasing worldwide, particularly in the low- and middle-income countries and especially among children and young adults [38]. According to the $\mathrm{WHO}$, the number of patients having asthma is about 300 million and it is expected to increase to 400 million by 2025. Patients with asthma and allergic diseases have a low quality of life [38].
Some studies suggested that the increased prevalence of allergic diseases may be developed due to lack of microbial stimuli during infancy and early childhood. Children with atopic diseases have different commensal bacterial groups in their intestines compared to non-atopic children, and such differences are also found between countries with high and low incidence of atopic diseases. To date, there is no enough evidence was detected that probiotics can treat or prevent allergic diseases in children [39-40]. The use of probiotics against eczema showed some benefit, but this is incompletely understood. A meta-analysis demonstrated that the use of probiotics showed efficacy in the reduction of eczema occurrence by $21 \%$, but its effects was limited to the first two years of life, and was lost by four years [3]. A study by Allen, et al. (2010) has reported that multi-strains probiotic administrated to pregnant women from 36 weeks of gestation till six months postpartum significantly improved atopic eczema, and-sensitivity to cow's milk and egg proteins in the newborn [18]. A recent study of Dennis-wall, et al. (2017) also found that the administration of multi-strain probiotics by adults has shown improvement in seasonal allergy, particularly the attacks of rhino-conjunctivitis and quality of life, the associated mechanism is still unclear, though it may be due to probiotics ability to increase regulatory $T$ cells and inducing tolerance [40]. However, the available evidence points that probiotics may have a role in allergy preventions though more research is needed to clarify this point.

\section{Cancer and probiotics}

Recently, the beneficial use of probiotics against cancer has been investigated because of their effects on inhibiting the growth of many harmful microorganisms that produce mutagens and carcinogens. A recent study by Motevaseli, et al. (2017) has discussed the effects of probiotics on the improvement 
of the immune system by protection from oxidative substance and other health benefits, like reducing the development of colorectal cancer (CRC) in animal models for example. The use of Lactobacillus rhamnosus $G G$ as a prophylaxis from CRC was found due to the induction of dimethyl hydrazine in animal models through different pathways [41, 42]. It was reported that the intestinal microflora of CRC patients were different from controls without cancer. The two most common observations were the increasing-variety of bacterial species and the abundance of specific strains like Peptostreptococcus, Selenomonas and Fusobacterium in CRC patients [43]. A change in the balance of the intestinal microflora could be a risk factor for the CRC by inhibiting the regulatory role of the normal microbial pattern, this effect may encourage the use of probiotics to decrease the severity of the CRC [43]. Sadahiro, et al. (2014) compared the use of probiotics and antibiotics in the preventions of surgical site infection, they found that probiotics did not differ from placebo [44], though a study by de Andrade Calaça, et al. (2017) reported that probiotics significantly prevented the infection and improved postoperative recovery in CRC patients [45].

\section{Probiotics and vaginal infection}

Vaginal infection is a common clinical problem in women, especially during the reproductive age. Bacterial vaginosis (BV) cause itching, pain and vaginal discharge. Treatment failure in BV results in the propagation of the aerobic intermediate flora and aerobic vaginitis (AV). The reduction of the vaginal commensal bacteria particularly Lactobacillus species that maintain the vaginal normal low pH produce bactericidal substances which compete with pathogens, and can induce over growth of anaerobic bacteria resulting in bacterial vaginosis (BV) [46]. The standard treatment with antibiotic for BV and AV has succeed in about 50\%. The oral use of probiotic containing three Lactobacillus strains for 10 days in association with antibiotic treatment for women with BV or AV demonstrated favorable effects in the prevention of recurrent symptoms in up to $51 \%$ compared to placebo [47]. Candida species commonly cause vulvovaginal candidiasis (VVC) in women, mostly married and pregnant, with symptoms profile similar to bacterial vaginitis [46]. VVC may be recurrent (rVVC) in up to $50 \%$ among treated women. In addition, to the recent high rates of vaginal candidiasis that demonstrates resistance to the treatment associated with the wide use of fluconazole [46]. The long-term use of vaginal probiotics capsules containing two Lactobacillus strains with antibiotic administration demonstrated significant prevention of rVVC for long time [47]. A study by Murina, et al. (2014) reported that the use of the long term two strains of Lactobacillus species as a vaginal slow release tablet has prevented the rVVC in $86 \%$ of patients for 10 weeks, and has also resulted in the inhibition of the rVVC for 7 months after discontinuing the probiotics in $72.3 \%$ of patients [48]. The same phenomena were demonstrated by $\mathrm{Ho}$, et al. (2016) in pregnant women whom were colonized with vaginal and rectal Group B Streptococcus, when they were treated with two Lactobacillus strains of probiotics, it was effective in $40 \%$ of women compared to $18 \%$ in the placebo group [49]. The oral administration of multi species probiotics has shown antimicrobial activity in vitro against Candida albicans and E. coli in pre-menopausal women and the ability of probiotics to prevent uro-genital infections and inhibit epithelial cell proliferation by increasing the vagina $\mathrm{pH}$ after 7 days [50-51]. All these results may encourage the production of genetically engineered probiotics which could be more efficient to prevent bacterial and yeast vaginal infection and might replace or reduce the administration of antibiotics in future [46].

\section{Other health benefits}

Commensal intestinal bacteria play a role in metabolism of proteins, lipids and carbohydrates. These 
bacteria ferment non-digestible carbohydrates that are not used by the host by production of $\mathrm{CO}_{2}, \mathrm{H}_{2}$, $\mathrm{CH}_{4}$ and short-chain fatty acids (SCFAs) including acetate, propionate and butyrate [52]. Most of these SCFAs are produced in the intestine and then absorbed by the host contributing to its energy. All data support the fact that SCFAs produced by the human gut microbiota are playing important roles as substrates for glucose, cholesterol, and lipids metabolism. Lactobacilli can produce SCFAs by the fermentation of carbohydrates end-products such as pyruvate, which is generated during the glycolytic pathway; and by the phosphoketolase route in a heterofermenting conditions. Bifidobacteria are using the fermentation pathway to produce mainly acetate and formate during growth under carbohydrates limitation, and acetate and lactate when carbohydrates are in excess. A recent study of Bermudez-Humaran and Langella has reported that genetically modified bacteria such as Bifidobacterium longum could produce many different effects, specially production of superoxide dismutase or catalase which has shown to cure colitis in mice. It is also found that Bifidobacterium species have selective migration to anaerobic sites of host tissues and can live in the hypoxic environment of the tumor [52]. A study by Brown, et al. (2004) has also demonstrated that the use of probiotic in normal subject can reduce the level of serum triglycerides by $27 \%$, and the probiotic composed of Lactobacillus reuteri_once administration in animal model has inhibited effectively hypercholesterolemia [9]. The production of SCFAs by these bacteria is potentially an essential regulatory effector of epithelial proliferation in the gut [52]. Many studies found that probiotics may decrease serum lipid level in humans and animal. The use of multi-strains probiotic in infant has clearly shown increase in SCFA in their fecal samples [53]. It was reported that certain strains of Lactobacilli and Bifidobacteria bacteria can produce B-vitamins (B1, $\mathrm{B} 2$, B12 or B9). Both of these bacterial groups were isolated from a variety of sources such as the human and animals gastrointestinal tract (GIT), dairy products, plants, and grains [54]. These vitamins are normally stored inside the cells and released by direct diffusion, using specific transporters in the cell membrane or via cellular lyses inside the GIT of the host, making these strains ideal candidates for the in-situ delivery of group B vitamins [54], like Lactobacillus fermentum (CECT5716) which was isolated from the lactating mother milk and has shown the ability to produce B2 and B9 in vitro [54].

\section{Safety}

Probiotics have been described generally safe (GRAS) by the US Food and Drug Administration (FDA). Their safety in human is established through consumption of probiotics in food and dairy products for long time, also, many probiotics bacteria species are already colonizing parts of human body [55-56]. However, both WHO and FAO stated that probiotics may results in several types of adverse events at least theoretically [9].

\section{Possible side effects}

\section{Systemic infections}

There are genuine concerns that the "bacterial" probiotics may cause infections like bacteremia, sepsis and endocarditis. Doron and Snydman reported from Finland that despite the increasing probiotics consumption over a decade, Lactobacillus bacteremia did not increase [9]. In France, the risk of Lactobacillus infection was estimated to be 1 per 10 million. Moreover, people living with HIV, premature infants, cancer patients on chemotherapy whom were given probiotics did not have any side effect [55]. Conversely, Sherid, et al. (2016) reported a case of liver abscess and bacteremia caused by Lactobacillus in 82-year-old female after using Lactobacillus-contain probiotic, but there was no con- 
firmation if the strain was related to the prescribed probiotic or a commensal bacterium [58]. Bacteremia with $L$. rhamnosus $G G$ in an adult patient was observed when he received an adjunctive probiotic treatment for ulcerative colitis [59]. Again, in a case report of Lactobacillus bacteremia in a 69-year-old male, there was no evidence of genetic relatedness of the used probiotic strain to the bacteremic strain as confirmed by three molecular methods [60]. All reported studies found rare occurrence of any side effects in association with probiotic treatment of patients.

\section{Adverse metabolic activity}

Some probiotics stains can produce D-lactate which could lead to acidosis. There is also concerns that probiotics may cause intestinal ischemia due to increasing demand of oxygen in the gut, especially in the critically ill patients with ischemic bowel diseases and compromised blood flow to the gut mucosa. Minor side effects like nausea, flatulence and abdominal cramps were reported, but most of the studies did not report any adverse reactions. Besides, one meta-analysis showed that there was a decrease of adverse events by $18-20 \%$ in patient who have received probiotics compared to control. WHO/FAO recommended to evaluate new probiotics strains safety by several methods including the presence of antibiotic resistance, hemolytic activity, ability to produce toxins, metabolic activities like D- lactate production in addition to consumers surveillance [9]. A recent study assessed the safety of Lactobacillus helveticus by several methods to assure its use as a probiotic. Its whole genome sequencing showed no antibiotic resistance gene that could be transferred, and there were no virulence factors and only few genes could result in adverse metabolite reactions. The study has also used rats to conduct acute oral toxicity tests, and has shown no mortality or toxicity and no significant change in weight, behavior and physical activity. The author concluded that their results suggested that use of Lactobacillus helveticus strain is safe for human consumption [56].

\section{Antibiotic resistance}

The administration of probiotics and their potential interaction with the human gut bacteria raise concerns about their antibiotic resistance profile and ability to transfer antibiotic resistance genes despite that not all resistance genes could be transferred in the host intestinal flora. Many factors can affect this transfer, such as genes persistence in plasmid or transposons and chromosomes, in addition, to the type of bacterial donors and recipients and the selective pressure of antibiotic administration [57]. However, the available methods can evaluate antibiotics resistance profile for bacteria. Genome sequencing could be used to asses both resistance genes and genes with the potential for adverse events [55]. Therefore, it is imperative to have knowledge of the probiotic resistance profile before its use with antimicrobials.

\section{Overstimulation of the immune system}

There are no studies that have shown that the consumption of probiotics could lead to autoimmune disease in humans, considering their effects on the innate immune systems.

\section{Conclusion}

Though probiotics have been used for decades, but the interest in their utilization as therapeutic, adjunctive and prophylactic remedies increased in recent years and have shown some benefits in several infections. Currently, the need arises for wellcontrolled studies on probiotics. Moreover, to study specific species, either as solitary or in combination in a variety of clinical conditions and demonstrate their health-related effects. 


\section{Acknowledgment}

This article is a part of a Master thesis project by Baraa Taha. which was supervised by Professor Drs. Asem A. Shehabi and Jamal Wadi, in the School of medicine, The University of Jordan, Amman, Jordan.

\section{References}

1. Markowiak P, Katarzyna Śliżewska K. Effects of Probiotics, Prebiotics, and Synbiotics on Human Health. Nutrients 2017; 9(9): 1021.

2. Doron S, Snydman D. Risk and safety of probiotics, Clin Infect Dis 2015; 60 (S2): S129-134.

3. West C Prescott S. Prebiotics and probiotics for prevention of allergic disease [Internet]. Diva-portal.org. 2017 [cited 8 February 2017].

4. Pineiro M, Asp NG, Reid G, Macfarlane S, Morelli L, Brunser O, Tuohy K. "FAO Technical meeting on prebiotics". J Clin Gastroenterol 2008; 42: 3, 2: S156-9.

5. Bermúdez-Humarán LG, Langella $P$. Use of traditional and genetically modified probiotics in human health: what does the future hold? Microbiol Spectr 2017; 5: (5) doi: 10.1128/ microbiolspec.BAD-0016-2016.

6. Scott K P, Antoine JM, Midtvedt T, van Hemert S. Manipulating the gut microbiota to maintain health and treat disease. Microb Ecol. Health Dis 2015; 26:25877.

7. Drisko Je, Giles C, Bischoff B. Probiotics in Health Maintenance and Disease Prevention. Altern Med Rev 2003; 8(2):143-155.

8. Nair SM, Amalaradjou MA, Venkitanarayanan K. Antivirulence Properties of Probiotics in Combating Microbial Pathogenesis. Adv Appl Microbiol 2017; 98, 1-29.

9. Brown A, Valiere A. Probiotics and medical nutrition therapy. Nutr Clin Care 2004; 7; (2): 56-68.

10. Singh VP, Sharma J, Babu S, Rizwanulla, Singla A. Role of probiotics in health and disease: A review, J Pak Med Assoc 2013; 63(2), 253-257

11. De Vrese M, Schrezenmeir J. Probiotics, prebiotics, and synbiotics. Adv Biochem Eng Biotechnol 2008; 111:1-66.

12. Hemarajata $P$, Versalovic J. Effects of probiotics on gut microbiota: mechanisms of intestinal immunomodulation and neuromodulation. Therap Advan Gastro 2013; 6(1):39-51.

13. He M. Shi B. Gut microbiota as a potential target of metabolic syndrome: the role of probiotics and prebiotics. Cell Biosci 2017; 7: 54.

14. Derwa Y, Gracie DJ, Hamlin PJ and Ford AC. Systematic review with meta-analysis: the efficacy of probiotics in inflammatory bowel disease. Aliment Pharmacol Ther 2017; 46:389-400.

15. Sears, Cynthia L. A dynamic partnership: celebrating our gut flora. Science 2005; 11(5): 247-251.
16. Walter J, Ley R. The human gut microbiome: ecology and recent evolutionary changes. Annu Rev Microbiol 2011; 65: 411-429.

17. Collado M, Meriluoto J, Salminen S. Role of commercial probiotic strains against human pathogen adhesion to intestinal mucus. Lett Appl Microbiol 2007; 45: 454-460.

18. Allen SJ, Martinez EG, Gregorio GV and Dans LF. Probiotics for treating acute infectious diarrhea. Cochrane Database of Systematic Reviews; 2010:11. Art. No.: CD003048.

19. McFarland Lynne. Probiotics and Diarrhea, Ann Nutr Metab 2010; 57(1):10-11.

20. Dubey AP, Rajeshwari K, Chakravarty A, Famularo G. Use of VSL\#3 in the Treatment of Rotavirus Diarrhea in Children, J Clin Gastroenterol 2008; 42(1),126-129.

21. Fox MJ, Ahuja KDK, Robertson IK, Ball MJ, Rajaraman E. Can probiotic yogurt prevent diarrhoea in children on antibiotics? A double-blind, randomised, placebo controlled study, BMJ Open 2015; , 5:e006474.

22. Ojetti V, laniro G, Tortora A, D'Angelo G, Rienze T, et al. The Effect of Lactobacillus reuteri Supplementation in Adults with Chronic Functional Constipation: a Randomized, Double-Blind, Placebo-Controlled Trial, J Gastrointestin Liver Dis 2014; 23 (4), 387-391.

23. Youngran $Y$, Jaejin L. Effect of a double-coated probiotic formulation on functional constipation in the elderly: a randomized, double blind, controlled study Arch Pharm Res2014; 38(7):1345-1350.

24. Liu Jin, Huang Xin-En. Efficacy of Bifidobacterium Tetragenous Viable Bacteria Tablets for Cancer Patients with Functional Constipation. APJCP 2014; 15 (23) 10241-10244.

25. Haghdoost M, Tahizadeh S, Montazer M, Poorshahverdi P, Ramouz A, Fakour S. Double strain probiotic effect on Helicobacter pylori infection treatment: A double-blinded randomized controlled trial. Caspian J Intern Med 2017; 8(3): 165-171.

26. SMC L, Ward A, Chamberlain RS. Probiotics improve the efficacy of standard triple therapy in the eradication of Helicobacter pylori: A meta-analysis. Infect Drug Resist 2016; 9:275-289.

27. Pakdaman M, Udani J, Jhanna M, Shahani M. The effects of the DDS-1 strain of Lactobacillus on symptomatic relief for lactose intolerance - a randomized, double-blind, placebo-controlled, crossover clinical trial. Nutr J 2016; 15:56.

28. Yoshimatsu Y, Yamada A, Furukawa Ri, Sono K, Osamura A, et al. Effectiveness of probiotic therapy for the prevention of relapse in patients with inactive ulcerative colitis, World J Gastroenterol 2015; 21(19): 5985-5994.

29. Fedorak R, Feagan B, Hotte N, Leddin D, Dieleman L, Petrunia $D$, et al. The Probiotic VSL\#3 Has Anti-inflammatory Effects and Could Reduce Endoscopic Recurrence After Surgery for Crohn's Disease. Clin Gastroenterol Hepatol 2015; 13(5), 928-935. 
30. Fujimori S, Tatsuguchi A, Gudis K, Kishida, Mitsui K, et al. High dose probiotic and prebiotic cotherapy for remission induction of active Crohn's disease, J Gastroenterolo Hepatolo 2006; 22, 1199-1204.

31. Ya-Hui $D$, Lin-Yan $Q$, Jie $P$, Jing-Yang $L$, Xu Qiang $X$, et al. The regulation of immune cells by Lactobacilli: a potential therapeutic target for anti-atherosclerosis therapy, Oncotarget 2017; 8( 35), 59915-59928.

32. Ashraf R, Shah N. Immune System Stimulation by Probiotic Microorganisms. Crit Rev Food Sci Nutr2014; 54:7, 938-956.

33. Zimmermann $P$, Curtis $N$. The influence of probiotics on vaccine responses - A systematic review. Vaccine 2018; 36: 2/4, 207213.

34. Jespersen L, Tarnow I, Eskesen D, Morberg C, Michelsen B, et al. Effect of Lactobacillus paracasei subsp. paracasei, L. casei 431 on immune response to influenza vaccination and upper respiratory tract infections in healthy adult volunteers: a randomized, double-blind, placebo-controlled, parallel-group study. Am J Clin Nutr 2015; 101,1188-1196.

35. Bosch M, Mendez M, Perez M, Farran A, Fuentes M, Cune J. Lactobacillus plantarum CECT7315 and CECT7316 stimulate immunoglobulin production after influenza vaccination in elderly, Nutricion Hospitalaria 2012; 27(2), 504-509.

36. Rizzardini G, Eskesen D, Calder P, Capetti A, Jespersen L, et al. Evaluation of the immune benefits of two probiotic strains Bifidobacterium animalis ssp. lactis, BB-12W and Lactobacillus paracasei ssp. paracasei, L. casei 431 w in an influenza vaccination model: a randomised, double-blind, placebo-controlled study. $\mathrm{Br}$ J Nutr 2012; 107, 876-884.

37. Singh VP, Sharma J, Babu S, Singla A. Role of probiotics in health and disease: A review, J Pak Med Assoc 2013; 63(2), 253-257.

38. World Allergy Organization (WAO) White Book on Allergy: Update 2013 Editors: Ruby Pawankar Giorgio Walter Canonica Stephen T. Holgate Richard F. Lockey Michael S. Blaiss). Copyright 2013 World Allergy Organization. www.worldallergy. org/UserFiles/file/WhiteBook 2-2013-v8.

39. Pan SJ, Kuo CH, Lam KP, Chu YT, Wang WL, et al.. Probiotics and allergy in children--an update review. Pediatr Allergy Immunol 2010; 21:e659.

40. Dennis-Wall JC, Culpepper T, Carmelo Nieves C, Rowe CC,3 Burns AM, et al. Probiotics (Lactobacillus gasseri KS-13, Bifidobacterium bifidum G9-1, and Bifidobacterium longum MM-2) improve rhinoconjunctivitis-specific quality of life in individuals with seasonal allergies: a double-blind, placebocontrolled, randomized trial. Am J Clin Nutr 2017; 105:758-67.

41. Motevaseli E, Dianatpour A, Ghafouri-Fard S. The Role of Probiotics in Cancer Treatment: Emphasis on their In Vivo and In Vitro Anti-metastatic Effects Int J Mol Cell Med Spring 2017; 6 (2), 66-76
42. Gamallat Y, Meyiah A, Kuugbee E, Hago A, Chiwala G, et al. Lactobacillus rhamnosus induced epithelial cell apoptosis, ameliorates inflammation and prevents colon cancer development in an animal model, Biomed Pharmacother 2016; 83, 536-54.

43. Hibberd AA, Lyra A, Ouwehand AC, Rolny $P$, Lindegren $H$, et al. Intestinal microbiota is altered in patients with colon cancer and modified by probiotic intervention. BMJ Open Gastro 2017; 4:e000145. doi:10.1136.

44. Sadahiro S, Suzuki T, Tanaka A, Okada K, Kamata H, et al. Comparison between oral antibiotics and probiotics as bowel preparation for elective colon cancer surgery to prevent infection: Prospective randomized trial. Surgery 2014; 155, 493503.

45. De Andrade Calaça PR, Bezerra RP, Albuquerque WWC, Porto ALF, Cavalcanti MTH. Probiotics as a preventive strategy for surgical infection in colorectal cancer patients: a systematic review and meta-analysis of randomized trials. Transl Gastroenterol Hepatol 2017; 23; 2:67.

46. Pendharkar S, Brandsborg $E$, Hammarström L, Marcotte $H$, Larsson P-G. Vaginal colonisation by probiotic Lactobacilli and clinical outcome in women conventionally treated for bacterial vaginosis and yeast infection, BMC Infect Dis 2015; 15, 255.

47. Heczko PB, Tomusiak A, Adamski P, Jakimiuk AJ, Stefański G, et al. Supplementation of standard antibiotic therapy with oral probiotics for bacterial vaginosis and aerobic vaginitis: a randomised, double-blind, placebo-controlled trial. BMC Women's Health 2015; 15,115.

48. Murina F, Graziottin A, Vicariotto F, Seta F. Can Lactobacillus fermentum LF10 and Lactobacillus acidophilus LAO2 in a Slowrelease Vaginal Product be Useful for Prevention of Recurrent Vulvovaginal Candidiasis? A Clinical Study. J Clin Gastroenterol 2014; 48, S102-S105.

49. Ho M, Chang YY, Chang WC, Lin HC, Wang MH, Lin, WC. et al. Oral Lactobacillus rhamnosus GR-1 and Lactobacillus reuteri RC-14 to reduce Group B Streptococcus colonization in pregnant women: A randomized controlled trial. Taiwan J Obstet Gynecol. 2016; 55:515-518.

50. Mezzasalma V, Manfrini E, Ferri E, Boccarusso M, Gennaro P, et al. Orally administered multispecies probiotic formulations to prevent uro-genital infections: a randomized placebo-controlled pilot study. Arch Gynecol Obstet (2017) 295: 527.

51. Matsuki T, Pedron T, Regnault B, Mulet C, Hara T, et al. Epithelial cell proliferation arrest induced by lactate and acetate from Lactobacillus casei and Bifidobacterium breve. PLoS One. 2013; 8:e63053.

52. Bermúdez-Humarán GL, Langella P. Use of Traditional and Genetically Modified Probiotics in Human Health: What Does the Future Hold? Microbiol Spect 2017; 5 no. 5. 
53. Kim H, Rutten N, Vaart Besseling, Niers L, Choi Y, Rijkers G, et al. Probiotic supplementation influences fecal short chain fatty acids in infants at high risk for eczema. Beneficial Microbes 2015; 6(6): 783-790.

54. LeBlanc JG, Milani C, de Giori GS, Sesma F, van Sinderen D, Ventura M. Bacteria as vitamin suppliers to their host: a gut microbiota perspective. Curr Opin Biotechnol 2013; 24:160-168.

55. Sanders ME, Akkermans LM, Haller D, Hammerman C, Heimbach J, et al. Safety assessment of probiotics for human use. Gut Microbes 2010; 1(3):164-185.

56. Bailiang L, Da J, Smith E, Na Li, Fenfen Y, et al. Safety Assessment of Lactobacillus helveticus KLDS1.8701 Based on Whole Genome Sequencing and Oral Toxicity Studies. Toxins 2017; 9, 301.

57. Allen SJ. The potential of probiotics to prevent Clostridium difficile infection. Infect Dis Clin North Am 2015; 29(1):135-144.

58. Sherid M, Samo S, Sulaiman $S$, Husein $H$, Sifuentes $H$ and Sridhar S. Liver abscess and bacteremia caused by lactobacillus: role of probiotics? Case report and review of the literature. BMC Gastroenterol 2016; 16(1):138.

59. Meini S, Laureano R, Fani L, Tascini C, Galano A, et al. Breakthrough Lactobacillus rhamnosus $G G$ bacteremia associated with probiotic use in an adult patient with severe active ulcerative colitis: case report and review of the literature. Infection 2015; 43(6):777-81.

60. Aroutcheva A, Auclair J, Frappier M, Millette M, Lolans K, de Montigny $D$, et al. Importance of molecular methods to determine whether a probiotic is the source of lactobacillus bacteremia. Probiot antimicrob proteins 2016; 8(1):31-40.

61. Brunser O. Probiotics: innocuousness, prevention and risks. Revista Chilena De Pediatria 2017; 88 (4), 534-540. 